\title{
OPTIMIZATION OF MATERIAL PROPERTIES OF PHASE CHANGE MATERIALS FOR LATENT HEAT THERMAL ENERGY STORAGE
}

\author{
Michal Březina ${ }^{a}$, Lubomír Klimeš ${ }^{b}$ and Josef Štětina ${ }^{a}$ \\ Brno University of Technology \\ ${ }^{a}$ Energy Institute, Department of Thermodynamics and Environmental Engineering \\ ${ }^{b}$ Sustainable Process Integration Laboratory (SPIL), NETME Centre \\ Technická 2896/2, 61669 Brno, Czech Republic \\ Michal.Brezina1@vutbr.cz, klimes@fme.vutbr.cz, stetina@fme.vutbr.cz
}

\begin{abstract}
Nowadays, people are increasingly interested in renewable energy sources and accumulation of energy for its efficient use. The use of non-renewable resources is progressively decreasing due to their adverse changes in climate conditions and high production of $\mathrm{CO}_{2}$ emissions. This work deals with the problem of heat accumulation by means of the phase change of a material using the Stefan problem, which serves to describe the temperature distribution in the medium and to determine the location of the interface between the solid and liquid phase. This approach is used to determine desired properties and thermal behaviour of the material under different accumulation requirements. The main objective was to create and solve an optimization model in order to determine heat transfer conditions and other parameters to ensure the extrema of thermal behaviour characterization.
\end{abstract}

Keywords: Stefan problem, phase change, phase change material (PCM), heat transfer, latent heat thermal energy storage (LHTES), optimization, GAMS

\section{Introduction}

Fossil fuels, which we have been used for decades, were formed in the Earths crust during millions or billions of years. The rate at which we use them will not make it available in a few hundred years, even with improved tapping methods. Our generation is also concerned about reducing the carbon foot print. Global warming and heat waves have brought the attention of investigators to search of new and renewable energy sources [1]. At the beginning of the 21st century, perceptions of climate change and energy consumption have changed dramatically. Mankind plays a dominant role in climate change on Earth, due to the production of a significant amount of $\mathrm{CO}_{2}$ in energy consumption, and it is therefore essential to reduce the amount of $\mathrm{CO}_{2}$ emissions released into the air in the near future. At the same time, the supply of energy in the form of fossil resources such as coal, oil became less reliable. In the spring of 2008 , the price of oil for the first time in history climbed up to 100 dollars per barrel. Nowadays, people have already accepted the idea of reducing fossil fuel consumption, and thus reducing $\mathrm{CO}_{2}$ emissions [2]. Therefore, renewable energy and its better use are at the forefront of the public's interest in achieving these goals. Thermal energy storage reservoirs play an important role in both heat and cold storage. Today, almost every household is equipped with a refrigerator, heating system, hot water facility and other appliances that use energy. The use of thermal energy storage covers a wide range of energy systems from centralized systems to autonomous regions and objects.

Thermal energy can be accumulated in the form of sensible heat, which uses the actual heating of the working substance and its specific heat capacity. For this type of accumulation, the most ideal substance appears to be water. Water is cheap, affordable and has a high heat capacity. However, it does not seem appropriate when it comes to the size requirements of storages and working temperature ranges. That is the reason why the latent heat accumulation is today becoming more and more important. This method of accumulation utilizes the thermal capacities of the substance and the phase transformation of the substance. Owing to this concept, the phase change material is able to accumulate more thermal energy while maintaining the same dimensions of the storage or to accumulate the same heat energy while reducing the storage size requirements [3].

Phase change materials (PCMs) are materials used in latent heat thermal energy storage. They can use both the latent and sensible to store and release heat. The substantial thermal energy transfer occurs when a material changes the state from one to another one. In practice, the change from solid to liquid and vice versa is dominantly utilized. Such materials are capable to absorb or release a large amount of heat in the process of hte phase change. A wide range of PCMs for various applications have been developed. Such materials differ in chemical composition as well as in thermal properties. In this work, the attention is aimed at the determination of optimal material properties for a heat accumulation application. The presented results are parts from the master thesis [3] of the pricipal author of the paper. 


\section{Thermal Accumulation}

Energy storage systems provide the potential to not only save the energy but also to reduce the environmental impact related to the energy use. In fact, these systems provide a valuable solution for the correction of the mismatch that is often present between the supply and demand of energy. Two main types of thermal storage exist. Sensible heat storage which use mostly water as an accumulation material and latent heat storage with a PCM, which are very attractive because of its high storage density within a small temperature range. Figs 1 and 2 show types of thermal energy storage and phase change materials, respectively.

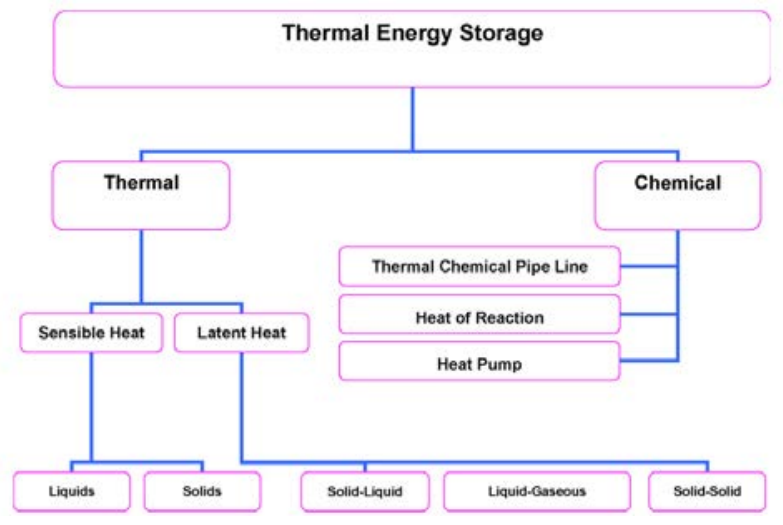

Figure 1: Thermal energy storage [4]

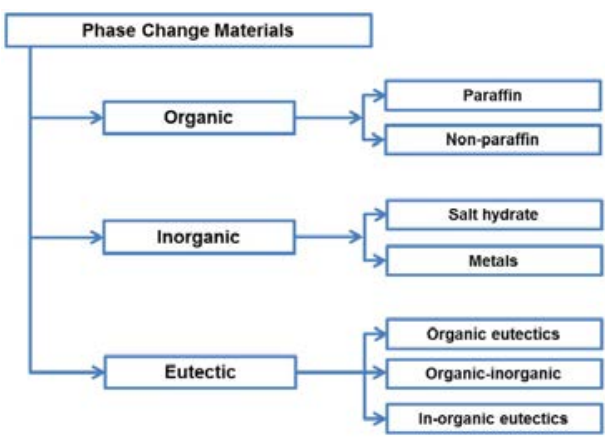

Figure 2: PCM classification [5]

\subsection{Thermal Energy Accumulation}

As mentioned above, energy storage facilities are mainly designed since the demand for energy does not always meet the actual amount of available energy. Thermal energy storage (see Fig. 1) can be applied with materials that use accumulation due to sensible heat, e.g. water or rocks, but also PCMs which employ the "extra" energy due to the phase change. In order to acquire this energy, it is necessary that this process is reversible [6]. In case of sensible heat accumulation (Fig. 3), only the thermal capacity of the substance within the given temperature range is used. The stored energy depends on the initial and final temperatures. The ratio of the stored heat $\Delta Q$ to the temperature rise $\Delta T$ is the heat capacity $C$ of the storage medium $\Delta Q=C \Delta T=m c \Delta T$. For the latent heat accumulation (Fig. 4), the accumulated heat energy is composed of sensible heat due to the temperature rise and of the latent heat due to the phase change of the material $\Delta Q=m \cdot c \cdot \Delta T+m \cdot l$, where $l$ is the specific latent heat. In the accumulation with the phase change of the material, PCM materials such as paraffins, salts hydrates, etc. are frequently used.

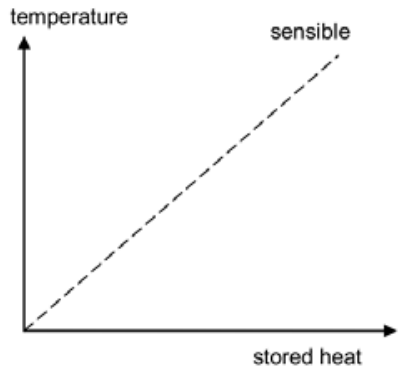

Figure 3: Heat storage in terms of sensible heat [2]

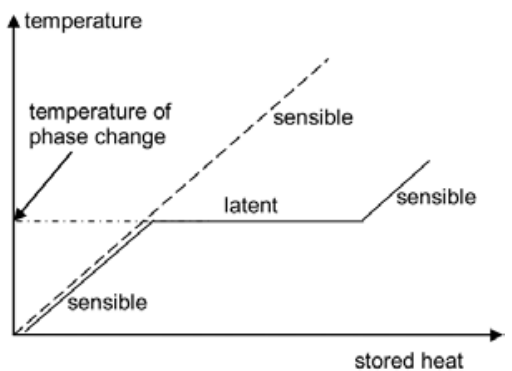

Figure 4: Heat storage in terms of latent heat [2]

\subsection{Phase Change Materials}

PCMs are able to store much more thermal energy per unit volume than sensible materials such as water, masonry, stones. It is known that properties of PCMs can be adapted to the desired applications. This is mainly done by means of their chemical composition [3]. In general, PCMs require for their use in thermal energy storage to exhibit the following thermodynamic, kinetic and chemical properties. Thermal properties: high latent heat of transition, high thermal conductivity, suitable melting/freezing temperature; Physical properties: small volume change, low vapor pressure, high density; Kinetic properties: sufficient crystallization rate, no supercooling; Chemical properties: long-term chemical stability, no toxicity, non-flammable, non-corrosive; Economics: cheap, cost effective, available, commercially viable. In order to achieve desired performance in terms of energy storage, the appropriate choice of a phase change material is necessary. 


\section{Mathematical Formulation}

\subsection{Stefan Problem}

The Stefan problem is a mathematical model for a phase change problem including the heat transfer equation and the phase interface which moves in time. The classical Stefan problem aims at the description of the temperature distribution in a homogenous body undergoing a phase change. This problem is solved via the heat transfer equation with a uniform initial temperature in the whole medium and with the Stefan boundary condition at the location of the interface between phases. The interface location is unknown, and this type of problem is called the free boundary problem. From the mathematical point of view, the phases are domains where the solution of the partial differential equation is continuous and sufficienty differentiable [3]. From a physical point of view, the solution represents the properties of each phase. The moving boundary is an infinitesimally thin surface which separates adjacent phases.

\subsection{Two Phase Problem of Solidification}

The exact solutions of phase change problems are confined to a few idealized situations. These are particularly the cases of a 1D infinite or semi-infinite domains with rather simple boundary conditions. The exact solutions are obtainable, if the problems permit a similarity solution [7], which enables two independent variables $x$ and $t$ to merge into a single similar variable $\frac{x}{\sqrt{t}}$.

In the paper we deal with the two-phase solidification problem. In this case we assume the solidification in a half-space where the solution of the temperature field in the liquid and solid phase is required. The liquid at the constant temperature $T_{i}$ greater than the melting temperature $T_{m}$ is at the time $t=0$ and position $x=0$ cooled down and maintained at the temperature $T_{0}$ for time $t>0$. This problem is displayed in Fig. 5 and its solution is known as the Neumann's solution.

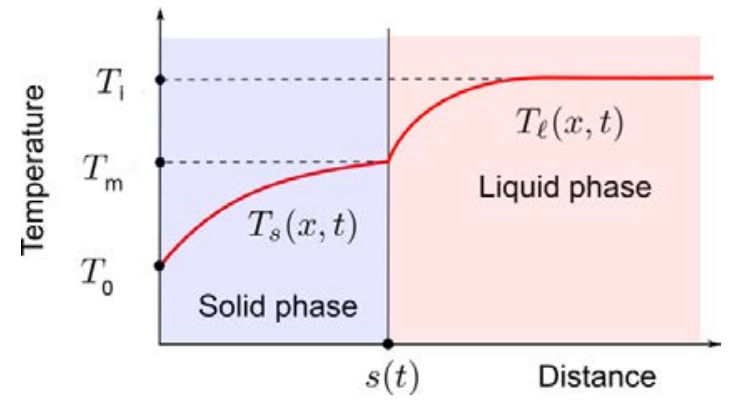

Figure 5: Two phase problem of solidification

Two phase problem has the following mathematical formulation [7]. The formulation for the solid phase is given as:

$$
\frac{\partial^{2} T_{s}(x, t)}{\partial x^{2}}=\frac{1}{\alpha_{s}} \frac{\partial T_{s}(x, t)}{\partial t} \quad \text { for } \quad 0<x<s(t), \quad t>0
$$

where $\alpha=\frac{k}{\rho c}$ is the thermal diffusivity and $s(t)$ is the position of the interface. At the boundary the Dirichlet's boundary condition $T_{s}(x=0, t)=T_{0}$ is applied. For the liquid phase the formulation is:

$$
\frac{\partial^{2} T_{l}(x, t)}{\partial x^{2}}=\frac{1}{\alpha_{l}} \frac{\partial T_{l}(x, t)}{\partial t} \quad \text { for } \quad s(t)<x<\infty, \quad t>0,
$$

with the initial condition $T_{l}(x>0, t=0)=T_{i}$ for the liquid phase and since we assume the semi-infinite body we also deal with conditions: $T_{l}(x \rightarrow \infty, t>0) \rightarrow T_{i}$, and the derivative is then considered to be $\left.\frac{\partial T}{\partial x}\right|_{x \rightarrow \infty}=0$. The coupling condition at the interface of solid and liquid phases is

$$
\left.k_{s} \frac{\partial T_{s}(x, t)}{\partial x}\right|_{x=s(t)}-\left.k_{l} \frac{\partial T_{l}(x, t)}{\partial x}\right|_{x=s(t)}=\rho L \frac{d s(t)}{d t}
$$

This heat transfer balance is called the Stefan condition, and $T_{s}(x=s(t), t)=T_{l}(x=s(t), t)=T_{m}$, corresponds to the phase change temperature at the interface [7]. With the use of all these assumptions, we get the timedependent location of the solid-liquid interface:

$$
s(t)=2 \lambda\left(\alpha_{s} t\right)^{\frac{1}{2}}
$$


where the $\lambda$ is the constant parameter. The temperature distribution of the solid and liquid phases can be calculated with the use of following equations [7]

$$
T_{s}(x, t)=T_{0}+\left(T_{m}-T_{0}\right) \frac{\operatorname{erf}\left[\frac{x}{2\left(\alpha_{s} t\right)^{\frac{1}{2}}}\right]}{\operatorname{erf}(\lambda)}
$$

and

$$
T_{l}(x, t)=T_{i}+\left(T_{m}-T_{i}\right) \frac{\operatorname{erfc}\left[\frac{x}{2\left(\alpha_{l} t\right)^{\frac{1}{2}}}\right]}{\operatorname{erfc}\left[\lambda\left(\frac{\alpha_{s}}{\alpha_{l}}\right)^{\frac{1}{2}}\right]} .
$$

In previous equations the Error function and its complementary Error function ${ }^{1}$ are used. The parameter $\lambda$ which appears in these equation is the root of the transcendental equation (TE). That TE can be obtained from the interface energy balance equation (3). We substitute the equations (4), (5) and (6) into (3) and with the use of relations $\frac{d s(t)}{d t}=\frac{\lambda \sqrt{\alpha_{l}}}{t^{\frac{1}{2}}}, \frac{d \operatorname{erfc}(z)}{d z}=-\frac{2}{\sqrt{\pi}} e^{-z^{2}}$ and $\frac{d \operatorname{erf}(z)}{d z}=\frac{2}{\sqrt{\pi}} e^{-z^{2}}$ we obtain the desired TE in the form

$$
\frac{e^{-\lambda^{2}}}{\operatorname{erf}(\lambda)}+\frac{k_{l}}{k_{s}}\left(\frac{\alpha_{s}}{\alpha_{l}}\right)^{\frac{1}{2}} \frac{T_{m}-T_{i}}{T_{m}-T_{0}} \frac{e^{-\lambda^{2}\left(\alpha_{s} / \alpha_{l}\right)}}{\operatorname{erfc}\left[\lambda\left(\alpha_{s} / \alpha_{l}\right)^{\frac{1}{2}}\right]}=\frac{\lambda L_{f} \sqrt{\pi}}{C_{s}\left(T_{m}-T_{0}\right)} .
$$

The solution of the TE can be obtained e.g. with the use of the numerical Newton method [3]. Once we calculate the parameter $\lambda$ from equation (7) we can determine the location of the solid-liquid interface $s(t)$ from Eq. (4), temperature field in solid phase $T_{s}(x, t)$ from Eq. (5), and in liquid phase $T_{l}(x, t)$ from Eq. (6).

\section{Optimization Approach}

For the optimization part of this work, we used GAMS. When designing optimization models, we focused on cases where we are looking for the thermal diffusivity $\alpha(k, \rho, c)$ and the latent heat $L_{f}$ in order to maximize or minimize the required heat transfer properties for specific cases. We can see that the problem is a problem of nonlinear programming, so we use the solver CONOPT to obtain the optimized properties of the model.

Nonlinear programming problem (NLP): Let $f: \mathbb{R}^{n} \rightarrow \mathbb{R}, g: \mathbb{R}^{n} \rightarrow \mathbb{R}^{m}$ a $X \subset \mathbb{R}^{n}$, and the symbol $\circ \in\{\leq, \geq,=\}^{m}$. Then

$$
\min _{\mathbf{x}}\{f(\mathbf{x}) \mid \mathbf{g}(\mathbf{x}) \circ \mathbf{0}, \mathbf{x} \in X\}
$$

is called the problem of nonlinear programming. A problem can arise while dealing with the equations where the Error function appears. The Error function has a slightly different mathematical formulation in MATLAB and GAMS and some mathematical modifications had to be done to calculate the Error functions.

\subsection{Problem Definition}

In this work, the main task is the optimization of heat transfer properties, which will extremize the desired thermal behavior. In case of the two phase solidification problem we calculate with these parameters: the cross section area $S=1\left[\mathrm{~m}^{2}\right]$, maximal time of accumulation $t_{\max }=1500[\mathrm{~s}]$, initial liquid temperature (constant in a whole medium for $t=0) T_{i}=340[\mathrm{~K}]$, phase change temperature $T_{m}=300[\mathrm{~K}]$ and the temperature for which the medium is cooled (for $x=0, t>0$ ) $T_{0}=270[\mathrm{~K}]$.

The material properties are financially valued, and therefore the material price constraints must be introduced. From practice we know that if we require $c, L_{f}$ and $k$ values to get higher, we will have to pay a higher price for the required properties, and therefore the price functions will increase. If we modify the density of the material, the price is the higher and the density of the body is smaller. When choosing price constraints, we used the above-mentioned facts and chose the limitations (9) which may be adjusted according to information from the manufacturer.

\footnotetext{
${ }^{1}$ In dealing with a following problem, we use the Error function erf $(x)$ defined as: $\operatorname{erf}(x)=\frac{1}{\sqrt{\pi}} \int_{-x}^{x} e^{-t^{2}} d t=\frac{2}{\sqrt{\pi}} \int_{0}^{x} e^{-t^{2}} d t$ And the complementary Error function erfc $(x): \operatorname{erfc}(x)=1-\operatorname{erf}(x)$.
} 


$$
\begin{aligned}
\operatorname{cost}_{k} & =1+e^{k} & & k \in\langle 0.05,2\rangle \\
\operatorname{cost}_{\rho} & =-0.0008 \cdot(\rho-450)+2.5 & & \rho \in\langle 450,1300\rangle \\
\operatorname{cost}_{c} & =2 \cdot 10^{-7} \cdot(c-600)^{2}+3 & & c \in\langle 1000,4000\rangle \\
\operatorname{cost}_{L_{f}} & =7 \cdot 10^{-11} \cdot L_{f}^{2}+0.01 \cdot e^{\frac{L}{5 \cdot 10^{4}}}+2 & & L_{f} \in\langle 50000,300000\rangle \\
\operatorname{cost}_{\text {total }} & \geq \operatorname{cost}_{k}+\operatorname{cost}_{\rho}+\operatorname{cost}_{c}+\operatorname{cena}_{L_{f}} & & \operatorname{cost}_{\text {total }} \in\langle 9.1,28.5\rangle
\end{aligned}
$$

The constraints (10) are introduced for the proper functionality of the model. The constant $a$ can be selected arbitrarily.

\subsection{Optimization Problems}

In the upcoming problems the optimization formulation is provided together with the graphical visualization of results gained by means of the solution of the problems in GAMS. The particular optimal values for material properties with the discussion are then summarized and presented in the chapter Results and Discussion in Table 1. We introduce four subproblems.

\subsubsection{Maximization of Interface Location}

The following mathematical formulation aims at the maximization of the interface position $s(t)$ so that it reaches the maximum position change $s\left(t_{\max }\right)$ from the beginning $s(0)=0$ for the desired time $t_{\max }$.

$$
\begin{gathered}
\operatorname{six}\left(t_{\max }\right)=2 \lambda \sqrt{\alpha_{s} t_{\max }} \\
\text { s.t. } \alpha_{l}=\frac{k_{l}}{\rho c_{l}}, \quad \alpha_{s}=\frac{k_{s}}{\rho c_{s}} \\
\frac{e^{-\lambda^{2}}}{\operatorname{erf}(\lambda)}+\frac{k_{l}}{k_{s}}\left(\frac{\alpha_{s}}{\alpha_{l}}\right)^{\frac{1}{2}} \frac{T_{m}-T_{i}}{T_{m}-T_{0}} \frac{e^{-\lambda^{2}\left(\alpha_{s} / \alpha_{l}\right)}}{\operatorname{erfc}\left[\lambda\left(\alpha_{s} / \alpha_{l}\right)^{\frac{1}{2}}\right]}=\frac{\lambda L_{f} \sqrt{\pi}}{C_{s}\left(T_{m}-T_{0}\right)} \\
+ \text { Limitations }(9) \text { and }(10)^{\lambda \in\langle 0.05,4\rangle[-], \text { Cost }_{\text {total }}=20\left[\text { Euro kg }{ }^{-1}\right],} \\
T_{i}=340[\mathrm{~K}], T_{m}=300[\mathrm{~K}], T_{0}=270[\mathrm{~K}], t_{\max }=1500[\mathrm{~s}] .
\end{gathered}
$$

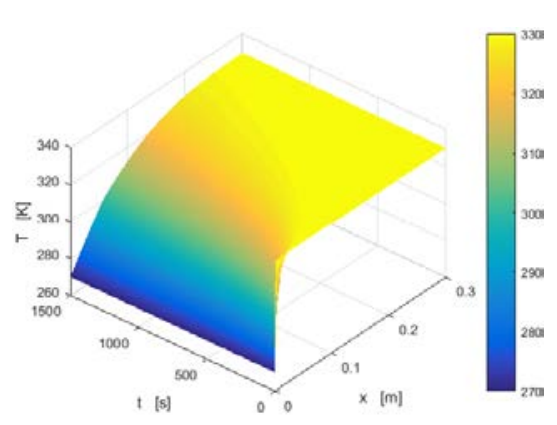

Figure 6: Temperature distribution for case 1 in $3 \mathrm{D}$

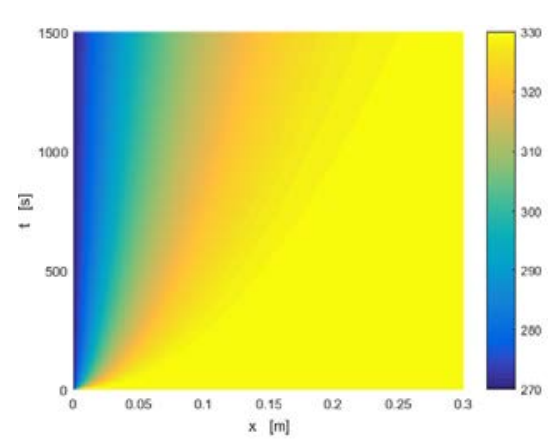

Figure 7: Temperature distribution for case 1 in $2 \mathrm{D}$

\subsubsection{Maximization of Boundary Temperature to Solidify Prescribed Region}

The aim is to find the maximum boundary temperature $T_{01}$ which leads to the solidification of the defined region of the melt given by $s_{r e q}$ in time $t_{\max }$. 


$$
\begin{gathered}
\max \\
\text { s.t. } \\
\alpha_{l}=\frac{k_{l}}{\rho c_{l}}, \alpha_{s}=\frac{k_{s}}{\rho c_{s}} \\
\frac{e^{-\lambda^{2}}}{\operatorname{erf}(\lambda)}+\frac{k_{l}}{k_{s}}\left(\frac{\alpha_{s}}{\alpha_{l}}\right)^{\frac{1}{2}} \frac{T_{m}-T_{i}}{T_{m}-T_{0}} \frac{e^{-\lambda^{2}\left(\alpha_{s} / \alpha_{l}\right)}}{\operatorname{erfc}\left[\lambda\left(\alpha_{s} / \alpha_{l}\right)^{\frac{1}{2}}\right]}=\frac{\lambda L_{f} \sqrt{\pi}}{C_{s}\left(T_{m}-T_{0}\right)} \\
s_{r e q}=0.025[\mathrm{~m}]+\text { Limitations }(9) \text { and }(10) \\
\lambda \in\langle 0.05,4\rangle[-], \text { Cost }_{\text {total }}=20\left[\text { Euro kg }^{-1}\right], T_{i}=340[\mathrm{~K}], \\
T_{m}=300[\mathrm{~K}], T_{0} \in\langle 255,295\rangle[\mathrm{K}], t_{\text {max }}=1500[\mathrm{~s}] .
\end{gathered}
$$

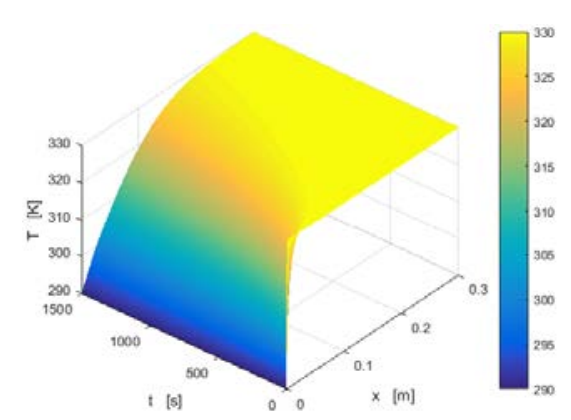

Figure 8: Temperature distribution for case 2 in $3 \mathrm{D}$

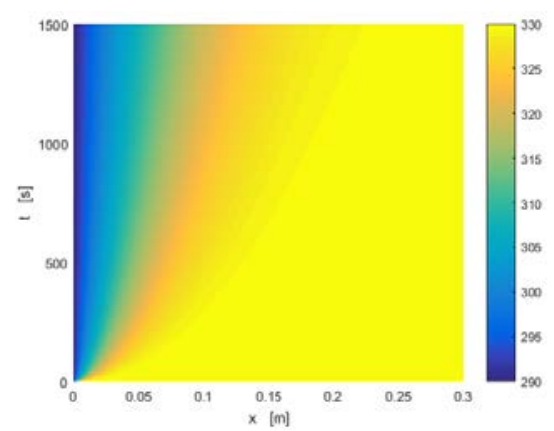

Figure 9: Temperature distribution for case 2 in $2 \mathrm{D}$

\subsubsection{Maximization of Heat Storage Flow Rate}

The problem is to maximize the average heat flow rate $\dot{Q}_{a v e}$ at the boundary. We also require that the location of the interface at the end of the time period $t_{\max }$ is equal to $s_{r e q}$.

$$
\begin{aligned}
& \max \quad \dot{Q}_{a v e}=\frac{Q}{t_{\max }} \\
& \text { s.t. } \quad Q=\sum_{t=1}^{t_{\max }} k S \frac{T_{m}-T_{0}}{\operatorname{erf}(\lambda)} \frac{e^{-x_{\text {surf }}^{2} /\left(4 \alpha_{s} t\right)}}{\sqrt{\pi \alpha_{s} t}} \\
& \alpha_{l}=\frac{k_{l}}{\rho c_{l}}, \alpha_{s}=\frac{k_{s}}{\rho c_{s}} \\
& \frac{e^{-\lambda^{2}}}{\operatorname{erf}(\lambda)}+\frac{k_{l}}{k_{s}}\left(\frac{\alpha_{s}}{\alpha_{l}}\right)^{\frac{1}{2}} \frac{T_{m}-T_{i}}{T_{m}-T_{0}} \frac{e^{-\lambda^{2}\left(\alpha_{s} / \alpha_{l}\right)}}{\operatorname{erfc}\left[\lambda\left(\alpha_{s} / \alpha_{l}\right)^{\frac{1}{2}}\right]}=\frac{\lambda L_{f} \sqrt{\pi}}{C_{s}\left(T_{m}-T_{0}\right)} \\
& s_{\text {req }}=0.025[\mathrm{~m}],+ \text { Limitations }(9) \text { and (10), Cost } \text { total }=20\left[\mathrm{Euro} \mathrm{kg}^{-1}\right] \\
& \lambda \in\langle 0.05,4\rangle[-], T_{i}=340[\mathrm{~K}], T_{m}=300[\mathrm{~K}], T_{0}=270[\mathrm{~K}], t_{\max }=1500[\mathrm{~s}] .
\end{aligned}
$$

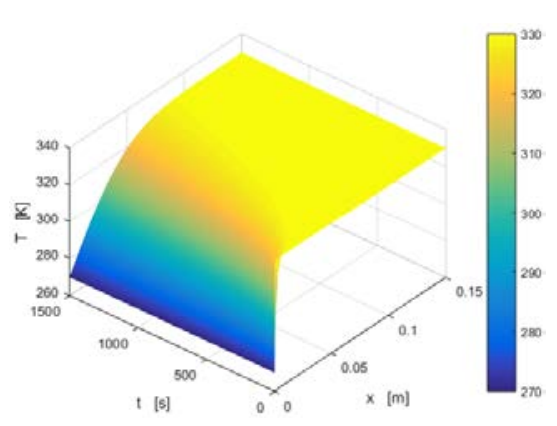

Figure 10: Temperature distribution for case 3 in 3D

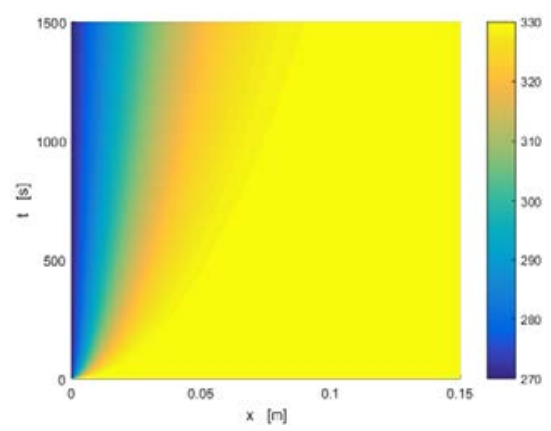

Figure 11: Temperature distribution for case 3 in $2 \mathrm{D}$ 


\subsubsection{Minimization of Total Cost of Material Properties}

The aim is to find the lowest total cost that a customer has to pay for the properties of material $\left(k, c, \rho, L_{f}\right)$ so that the heat energy released over time $t_{\max }$ is equal to the required released energy $Q_{r e q}$ using at most $s_{\max }$ interface location of the material.

$$
\begin{gathered}
\text { Cost }_{\text {total }}=\text { cost }_{k}+\operatorname{cost}_{\rho}+\operatorname{cost}_{c}+\text { cost }_{L_{f}} \\
\text { s.t. } \\
Q=\sum_{t=1}^{t_{\text {max }}} k S \frac{T_{m}-T_{0}}{\operatorname{erf}(\lambda)} \frac{e^{-x_{\text {surf }}^{2} /\left(4 \alpha_{s} t\right)}}{\sqrt{\pi \alpha_{s} t}} \\
Q=Q_{r e q}, s\left(t_{\max }\right) \leq s_{\max } \\
\frac{e^{-\lambda^{2}}}{\operatorname{erf}(\lambda)}+\frac{k_{l}}{k_{s}}\left(\frac{\alpha_{s}}{\alpha_{l}}\right)^{\frac{1}{2}} \frac{T_{m}-T_{i}}{T_{m}-T_{0}} \frac{e^{-\lambda^{2}\left(\alpha_{s} / \alpha_{l}\right)}}{\operatorname{erfc}\left[\lambda\left(\alpha_{s} / \alpha_{l}\right)^{\frac{1}{2}}\right]}-\frac{\lambda L_{f} \sqrt{\pi}}{C_{s}\left(T_{m}-T_{0}\right)}=0 \\
\alpha_{l}=\frac{k_{l}}{\rho c_{l}}, \alpha_{s}=\frac{k_{s}}{\rho c_{s}}, \lambda \in\langle 0.05,4\rangle[-],+ \text { Limitations }(9) \text { and }(10) \\
T_{i}=340[\mathrm{~K}], T_{m}=300[\mathrm{~K}], T_{0}=270[\mathrm{~K}], t_{\text {max }}=1500[\mathrm{~s}], Q_{r e q}=10[\mathrm{MJ}], s_{\max }=0.2[\mathrm{~m}]
\end{gathered}
$$

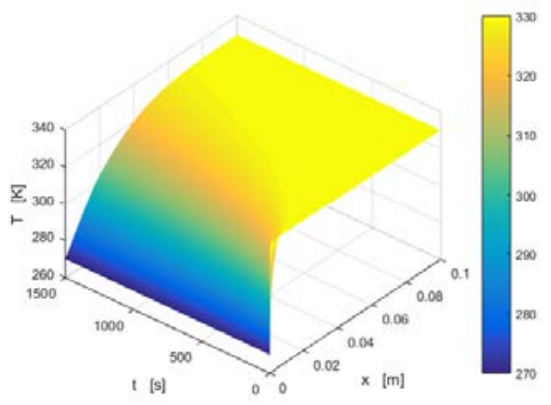

Figure 12: Temperature distribution for case 4 in $3 \mathrm{D}$

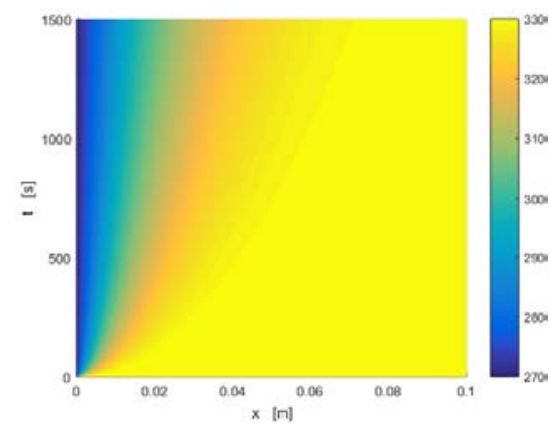

Figure 13: Temperature distribution for case 4 in $2 \mathrm{D}$

\section{$5 \quad$ Results and Discussion}

Summarized results for each optimization problem are displayed in Table 1. For all the optimization problem the optimal values were found. In the first case, we maximize the location of the interface $s(t)$. In the second case we request the maximum boundary temperature which ensures the solidification of the prescribed region occupied with the melt. In other two cases we deal with the requirement to maximize the heat flow and to minimize the price for PCM production in order to satisfy the request for the heat withdrawal.

Case 1: While maximizing the interface location the material properties reached the assumed values. The density $\rho$, the specific heat capacities in both the phases $c_{s}, c_{l}$ and the latent heat $L_{f}$ are at the minimum of the possible values. While the thermal conductivity $k_{s}$ is at its maximum, the thermal conductivity in the liquid phase $k_{l}$ reached the maximum as well, but due to the limitation (10) the value is smaller than $k_{s}$.

Table 1: Summarized results of the optimization cases

\begin{tabular}{c|cccc||c|cccc}
\hline Param & Case 1 & Case 2 & Case 3 & Case 4 & Param & Case 1 & Case 2 & Case 3 & Case 4 \\
\hline \hline$k_{s}$ & 2. & 1.684 & 2.0 & 1.848 & $\lambda$ & 0.346 & 0.176 & 0.436 & 0.369 \\
$k_{l}$ & 1.7 & 1.431 & 1.7 & 1.571 & $\alpha_{s}$ & $4.444 \mathrm{E}-6$ & $3.371 \mathrm{E}-6$ & $5.472 \mathrm{E}-7$ & $3.554 \mathrm{E}-7$ \\
$\rho$ & 450 & 491 & 913 & 1300 & $\alpha_{l}$ & $3.778 \mathrm{E}-6$ & $2.865 \mathrm{E}-6$ & $4.651 \mathrm{E}-7$ & $3.021 \mathrm{E}-7$ \\
$L_{f}$ & 50000 & 50637 & 50000 & 148590 & $s$ & 0.056 & 0.025 & 0.025 & 0.017 \\
$c_{s}$ & 1000 & 1016 & 4000 & 4000 & $Q_{\text {ave }}$ & - & - & 5010 & 6666 \\
$c_{l}$ & 1000 & 1016 & 4000 & 4000 & $Q_{\text {rel }}$ & - & - & $7.516 \mathrm{E}+6$ & $1 \mathrm{E}+7$ \\
\hline cost $_{k}$ & 7.432 & 5.784 & 7.432 & 6.581 & cost $_{L_{f}}$ & 2.202 & 2.207 & 2.202 & 3.741 \\
cost $_{\rho}$ & 2.500 & 2.467 & 2.129 & 1.820 & cost $_{\text {total }}$ & 15.17 & 13.49 & 17.07 & 17.45 \\
cost $_{c}$ & 3.035 & 3.035 & 5.312 & 5.312 & $T_{01}$ & - & 290 & - & - \\
\hline
\end{tabular}

Case 2: This case deals with the determination of the maximum boundary temperature which ensures the solidification of the prescribed region of the melt. All these conditions are satisfied and the temperature $T_{01}$ 
reaches its maximum value from the interval. The price for the total PCM production is at its lowest price of all the other optimization cases.

Case 3: In case of the maximum heat flow the thermal conductivities play the most important role because of the Fourier's law and those are at their maximum values. The condition of the interface location has to be satisfied and that is why the specific heat capacity is at its maximum value because it has to compensate that maximum thermal conductivity. It means that the heat dissipates more quickly and for the required interface location we have to use a material with a higher accumulation capacity to slow down the heat dissipation. Because the model modifies primarily the properties having smaller costs, the latent heat is at its minimum value due to its price and due to the fact that the condition was satisfied with the specific heat capacity and with the increasing density.

Case 4: The optimization task for the minimum total cost of the material properties was barely accomplished. If we look into Table 1 we can see that the total cost is the highest from all of the optimization cases. Nevertheless, the price for the material is the smallest possible subjected to all the constraints. This is mainly caused due to the condition of the required heat withdrawal $Q_{r e q}$. This condition is satisfied as well as the modified condition of the required interface location which is in this case in the form of two inequalities rather than in one equality constraint. The density and the specific heat capacity are at their maximum values. The latent heat was almost at its minimum value because the price for the material with the higher latent heat has the highest price from all of the properties.

\section{Conclusion}

The Stefan problem with the heat transfer equation was studied and utilised in optimization of different types of optimization of material properties. Optimal results have been achieved in all optimization cases. In some cases, it is necessary to fulfill rather strict constraints and we need to handle these constraints properly in order to solve the model. These problematic conditions were alleviated by the replacement of the equality formulation with the inequality formulations. In cases 3 and 4 the obtained results may be misleading at the very first sight. But if we consider the Fourier's law, we can say that if we want a higher heat flow, we have to increase the thermal conductivity. But the higher thermal conductivity means that the heat has to be transferred through a large amount (thickness) of the material and the heat flow is therefore decreasing. That is the reason why the optimal material in case 4 needs a higher value of the heat capacity to assure a higher heat withdrawal.

Acknowledgement: The authors gratefully acknowledge funding from the Ministry of Education, Youth and Sports of the Czech Republic under the National Sustainability Programme I (Project LO1202 NETME CENTRE PLUS) and the project Sustainable Process Integration Laboratory_SPIL, No. CZ.02.1.01/0.0/0.0/15_003/ 0000456, funded by European Research Development Fund, Czech Republic Operational Programme Research, Development and Education, Priority 1: Strengthening capacity for quality research.

\section{References}

[1] Ibrahim, N.I., Al-Sulaiman, F.A., Rahman, S., Yilbas, B.S., Sahin, A.Z.: Heat transfer enhancement of phase change materials for thermal energy storage applications: A critical review. Renew. Sustain. Energy Rev. 74(October 2015), 26-50 (2017).

[2] Mehling, H., Cabeza, L.F.: Heat and cold storage with PCM: an up to date introduction into basics and applications. Berlin: Springer-Verlag, (2008). ISBN 978-3-540-68556-2.

[3] Březina, M.: Optimization of a Stefan problem with heat conduction and phase change., Brno University of technology, Faculty of mechanical engineering., (Master thesis in CZE), 81 p., Supervisor: Lubomír Klimeš, (2017).

[4] Sharma, A., Tyagi, V.V., Chen, C.R., Buddhi, D.: Review on thermal energy storage with phase change materials and applications. Renew. Sustain. Energy Rev. 13(2), 318-345 (2009).

[5] Mohamed, S.A., et al.: A review on current status and challenges of inorganic phase change materials for thermal energy storage systems Renew. Sustain. Energy Rev. 70(December 2016), 1072-1089 (2017).

[6] Agyenim, F., Hewitt, N., Eames, P., Smyth M.: A review of materials, heat transfer and phase change problem formulation for latent heat thermal energy storage systems (LHTESS). Renew. Sustain. Energy Rev. 14(2), 615-628 (2010).

[7] Hahn, D. W. and Özişik, M.N.: Heat conduction. third edn. Hoboken: Wiley, (2012). ISBN 9780470902936. 